\title{
Treatment-Emergent Mutations and Resistance in HIV-Infected Children Treated with Fosamprenavir-Containing Antiretroviral Regimens
}

\author{
Lisa L. Ross ${ }^{*}, 1$, Mark F. Cotton ${ }^{2}$, Haseena Cassim ${ }^{3}$, Eugeny Voronin ${ }^{4}$, Naomi Givens ${ }^{5}$, Jorg Sievers ${ }^{5}$, \\ and Katharine Y. Cheng ${ }^{5}$ For the APV29005 \& APV20002 Pediatric Study Groups \\ ${ }^{1}$ ViiV Healthcare, Research Triangle Park, NC, USA \\ ${ }^{2}$ Department of Pediatrics and Child Health, Children's Infectious Diseases Clinical Research Unit, Stellenbosch \\ University, Tygerberg, South Africa \\ ${ }^{3}$ Perinatal HIV Research Unit, University of the Witwatersrand, Johannesburg, South Africa \\ ${ }^{4}$ Republic Hospital of Infectious Disease, St. Petersburg, Russian Federation \\ ${ }^{5}$ GlaxoSmithKline, Stockley Park, UK
}

\begin{abstract}
Treatment-emergent mutations and drug resistance were analyzed in virus from HIV-infected children meeting virologic failure (VF) criteria over 48 weeks following treatment with unboosted fosamprenavir or fosamprenavir/ritonavir-containing regimens in studies APV20002 and APV29005. Both antiretroviral therapy (ART)naïve and ART-experienced patients were enrolled. Patients met VF criteria by either failing to suppress HIV-RNA to $<400$ copies $/ \mathrm{mL}$ through week 24 or after confirmed viral rebound ( $\geq 400$ copies $/ \mathrm{mL}$ ) anytime through week 48 . Viral isolates were analyzed for treatment-emergent mutations or reduced drug susceptibility. Through week 48, 25/109 (23\%) of APV29005 and 9/54 (17\%) APV20002 study patients met VF. VF was more common in ART-experienced patients (68\% and $78 \%$, respectively). Major or minor treatment-emergent mutations were detected at VF in virus from 3 patients receiving unboosted fosamprenavir-containing regimens and in virus from 10 patients receiving fosamprenavir/ritonavircontaining regimens across the two studies. Major protease inhibitor mutations and the reverse transcriptase mutation M184V were detected at VF in virus from 4 and 5 patients, respectively, across both studies. Reduced drug susceptibility to any drug emerged in virus from 9 patients at $\mathrm{VF}$, although reduced fosamprenavir susceptibility emerged in virus from only 4 patients (2 ART-naïve and 2 ART-experienced). No cross-resistance to the protease inhibitor darunavir was observed.

In conclusion, given the high proportion of ART-experienced children (71\%) in these two studies, the overall incidence of children meeting VF criteria through 48 weeks was relatively low (21\%) and development of fosamprenavir reduced drug susceptibility at VF was uncommon, further supporting the use of fosamprenavir-containing ART regimens in HIVinfected children.
\end{abstract}

Keywords: Children, fosamprenavir, mutation, pediatric, resistance.

\section{INTRODUCTION}

Human immunodeficiency virus (HIV) infection remains a major public health concern. HIV protease is essential for the generation of mature infectious virus particles and is crucial for the HIV life cycle. Protease inhibitors are used in potent combination antiretroviral therapy (ART) to inhibit viral replication in HIV-infected patients. The efficacy, safety, and resistance profile of fosamprenavir (FPV), the phosphate ester pro-drug of the protease inhibitor (PI) amprenavir, has previously been evaluated in HIV-infected ART-naïve and ART-experienced adults [1-5].

*Address correspondence to this author at the ViiV Healthcare Clinical Sciences Group, 5 Moore Drive, Research Triangle Park, NC 27709, USA; Tel: 919-483-6325; E-mail: lisa.1.ross@viivhealthcare.com
While the FPV resistance profile is well documented in HIV-infected adults, there have been few previous pediatric studies utilizing FPV-based regimens and these have had with very limited resistance data [6, 7]. When HIV-infected children experience VF while receiving FPV-containing ART regimens as first or second line or salvage therapy, it is vital to know if the FPV resistance-associated mutations that can be selected will be the same as those seen in the more frequently studied adult population and whether these mutations result in cross resistance to other PIs and thus impact future drug sequencing options. It is also important to enroll both children infected with non-B HIV-1 subtypes in addition to those infected with $B$ subtypes in clinical studies to better understand if subtype influences FPV mutation selection.

APV20002 was an international, 48-week, Phase II, open-label, 2-cohort, multicenter study conducted in HIV-1 
infected pediatric subjects 4 weeks to $<2$ years old and was designed to define the FPV given twice-daily (BID) and FPV/ ritonavir (FPV/RTV) BID dosage regimens that would provide steady state amprenavir exposure similar to those observed in adults who received unboosted FPV-containing regimens or FPV/RTV-containing regimens. The APV29005 study was similarly designed to evaluate unboosted FPV BID-containing regimens and FPV/RTV BID-containing regimens in pediatric subjects aged 2 to 18 years. The 48 week pharmacokinetics (PK), safety and antiviral activity data from these studies have recently been published $[8,9]$ and included a brief summation of the overall resistance findings. Here we further describe the HIV subtypes represented in the study populations, and provide a more detailed and comprehensive analysis of the virologic failure (VF) populations, including virologic response profiles for individual study patients meeting VF criteria which further describe prior treatment status, specific concomitant antiretrovirals, and the pattern of selection of drug resistance mutations and reduced drug susceptibility that emerged over time in these patients.

\section{METHODS}

Study methods and design for both trials were described previously [8, 9]. Briefly, APV29005 (ClinicalTrials.gov NCT00089583) was an international, Phase II, open-label, multi-cohort, multicenter study that enrolled HIV-1-infected, ART-naïve or -experienced children from 2-18 years with screening plasma HIV-1 RNA $\geq 400$ copies $/ \mathrm{mL}$ at 30 sites in North America, Europe and South Africa [8]. Patients aged 2 to $<6$ years received either unboosted FPV (allowed only for PI-naïve patients) or FPV/RTV BID, while those aged $\geq 6$ years received FPV/RTV BID. FPV was administered as either $50 \mathrm{mg} / \mathrm{mL}$ oral suspension or $700 \mathrm{mg}$ tablets (permitted for children $>39$ kilograms). RTV was given either as an $80 \mathrm{mg} / \mathrm{mL}$ oral solution or as $100 \mathrm{mg}$ capsules (permitted for children $>33$ kilograms). Background ART included 2 or 3 nucleoside reverse transcriptase inhibitors (NRTIs).

APV20002 (ClinicalTrials.gov NCT00071760) was an international, Phase II, open-label, two-cohort, multicenter study that enrolled HIV-1 infected, ART-naïve or experienced children aged 4 weeks to $<2$ years from 7 sites in South Africa, Mexico, Argentina and Portugal with screening plasma HIV-1 RNA $\geq 400$ copies/mL [9]. Study patients received FPV/RTV (both weight- and age-based dosing were used for the oral liquid formulations) plus 2 NRTIs. Both studies were conducted in accordance with the Declaration of Helsinki and relevant national and institutional standards and approved by the Institutional Review Board or Ethics Committee for each site, with written informed consent obtained from the parent or legal guardian of all participating children.

Patients with $<1$ week's treatment with any PI were considered PI-naïve. Eligible PI-experienced patients had $>1$ week of exposure to $\leq 3 \mathrm{PIs}$, although prior use of low-dose RTV was not considered PI exposure. In the virology analyses described here, VF was defined as failure to achieve plasma HIV-1 RNA $<400$ copies $/ \mathrm{mL}$ by week 24 or a confirmed HIV-1 RNA rebound to $\geq 400$ copies $/ \mathrm{mL}$ after achieving HIV-1 RNA $<400$ copies $/ \mathrm{mL}$. Subjects meeting
VF criteria were not required to withdraw from the study. HIV-1 genotypic, subtype and phenotypic analysis (Monogram BioSciences, South San Francisco, CA) was attempted for baseline and VF timepoint samples for all subjects meeting VF criteria. Some samples yielded no results, and if no results were obtained, analysis of an additional sample collected with HIV-RNA $>400 \quad \mathrm{c} / \mathrm{mL}$ within 3 months of initial VF was also attempted. Analysis of additional baseline samples was performed as requested by site physicians to guide treatment decisions. Treatmentemergent resistance-associated mutations were defined by IAS-USA guidelines [10].

\section{RESULTS}

\section{APV29005 and APV20002 Study Populations at Baseline}

A total of 163 children were enrolled and initiated treatment across the two clinical studies. These included 109 children enrolled in Study APV29005 and 54 children enrolled in Study APV20002.

Of the 109 children aged 2-18 years in study APV29005 who received $\geq 1$ dose of study medication, $37 \%$ (40/109) had previously been exposed to one or more PIs. Nelfinavir was the most commonly prescribed prior PI $(33 / 40,83 \%)$. The median duration of prior PI exposure was 225 weeks. Sixty-two percent $(68 / 109)$ of the study patients were ARTexperienced prior to study entry, although within the cohort of patients who received unboosted FPV-containing regimens (which was restricted to patients with no prior PI treatment), 90\% (18/20) patients were ART-naive. Prior NRTI and NNRTI ART taken by the ART-experienced patients included abacavir, didanosine, efavirenz, emtricitabine lamivudine, nevirapine stavudine, tenofovir, zalcitabine, and zidovudine. Of the 54 enrolled patients from study APV20002, all were $<2$ years of age at the start of the study. Sixteen $(30 \%)$ patients were ART-naïve at the study start. The majority of patients $(61 \% ; 33 / 54)$ were ARTexperienced but were PI-naïve. Five $(9 \%)$ patients were ART and PI-experienced. Their prior PI exposure included nelfinavir, lopinavir/RTV or high dose RTV only. The median duration of prior PI exposure was 39 weeks. Prior NRTI or NNRTI ART for ART-experienced patients included abacavir, lamivudine, didanosine, stavudine, zidovudine and nevirapine.

\section{HIV-1 Subtype Analysis}

While pre-therapy/baseline HIV genotyping was not required for enrollment in the APV20002 and APV29005 studies, site physicians could request this analysis to help guide ART choices. Baseline HIV genotypic analysis was also attempted for any subject who met VF criteria. HIV-1 subtype data was obtained from 96/163 patients (Table 1). Of these, $50 \%$ of the HIV-1 group M subtypes analyzed from the two study populations were subtype B and 50\% were non-B; these included subtypes A, C, F, G and chimeric/complex. The subtype representation was fairly broad and reflected the inclusion of study recruitment sites in countries where non-B subtypes are common. When analyzed by study, several different subtypes or circulating recombination forms were represented in virus in the 
Table 1. The HIV-1 subtypes obtained from patients in the APV29005 and APV20002 Studies Virology Baseline population.

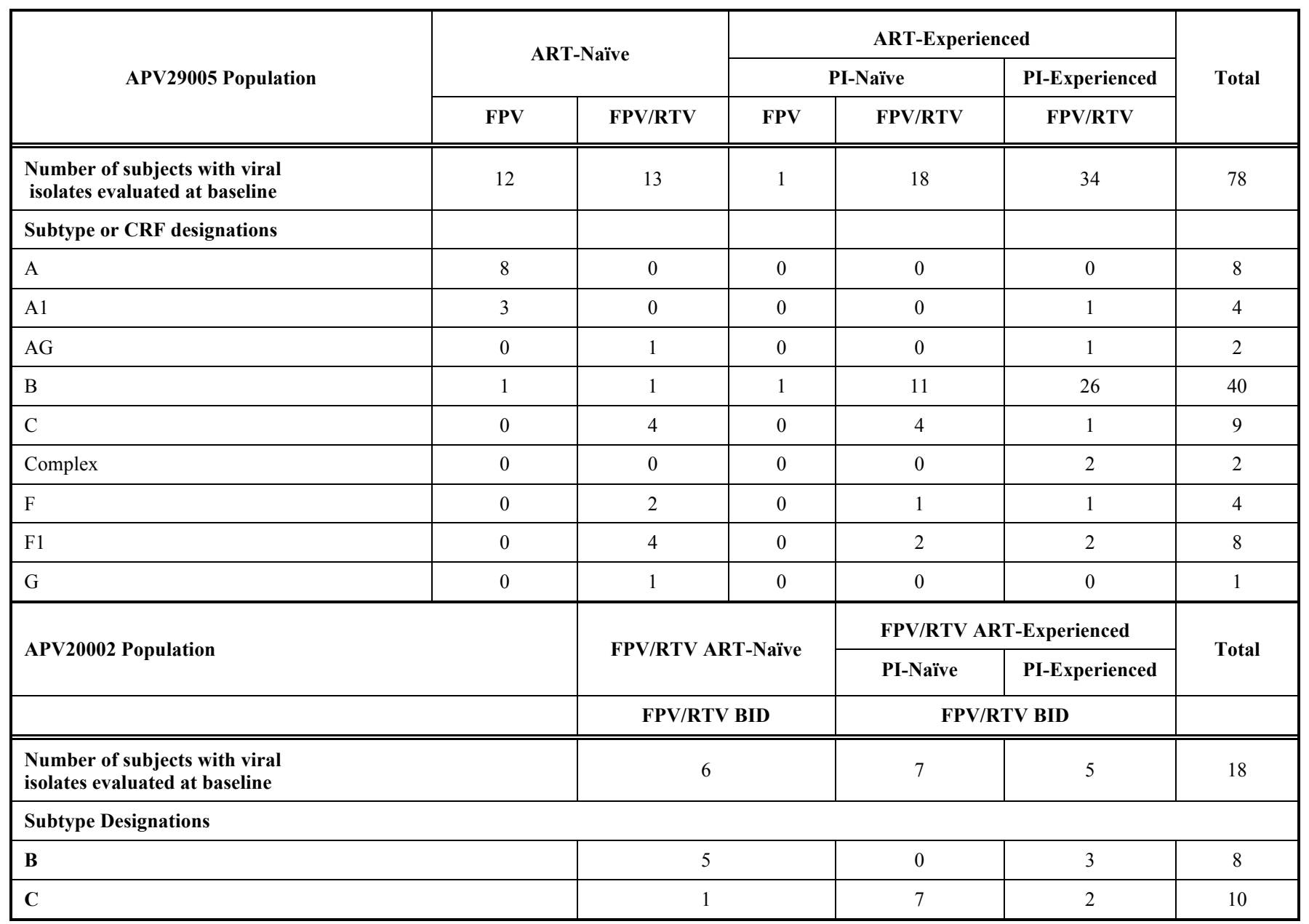

enrolled patients, including 38 non-subtype B viruses for patients in study APV29005. For study APV20002, subtypes $\mathrm{B}$ and $\mathrm{C} \mathrm{HIV-1}$ were the only two subtypes detected, with subtype $\mathrm{C}$ being slightly more prevalent $(56 \%)$.

\section{APV29005 Study VF Population}

VF criteria were met though 48 weeks by $25 / 109$ patients (23\%; Table 2 ). Of these, 8 patients $(32 \%)$ were previously ART-naïve and $17(68 \%)$ were ART-experienced. In the ART-naïve group, 6/8 patients with VF had received unboosted FPV-containing regimens while two had received FPV/RTV-containing regimens. All of the the ARTexperienced patients with VF had been treated with FPV/RTV-containing regimens. Of these, four patients had no prior PI exposure while the remaining 13 patients had prior PI exposure. Phenotypic drug susceptibility scores were available at baseline for $21 / 25$ patients with VF. Ten of these patients with VF $(48 \%)$ had been placed onto regimens containing $\leq 2$ phenotypically active drugs.

\section{APV20002 Study VF Population}

VF criteria were met by $9 / 54$ patients (17\%). Two were ART-naïve at study start, five were ART-experienced but were PI-naïve, and two were ART-experienced and also had prior PI exposure. Among the patients with VF, the median baseline CD4 cell percentage was $20 \%$; and the CD4+ cell count 937 cells $/ \mathrm{mm}^{3}$ (Table 2). The CD4+ cell percentages and actual cell counts in the VF population were slightly lower than what was observed in the overall study population $\left(26 \% ; 1235 \mathrm{cell} / \mathrm{mm}^{3}\right)$ [8].

\section{Treatment-Emergent HIV-1 Mutations and RS in the VF Population in Study APV29005}

The majority of patients meeting VF criteria (15/25 patients; $60 \%$ ) had paired HIV genotypes available for comparison at both baseline and at VF timepoints. Of these, $47 \%(7 / 15)$ of the patients with paired baseline and VF timepoint viral genotypes had virus with treatment-emergent resistance mutations. Of these, three were from patients receiving unboosted FPV-containing regimens and four were from patients receiving FPV/RTV-containing regimens (Table 3).

All three of the FPV-treated patients with VF were ARTnaïve at baseline. At VF, all three had HIV-1 with the treatment-emergent reverse transcriptase (RT) mutation M184V (individual response profiles are shown in Supplementary Fig. 1). Virus from these three patients developed reduced susceptibility (RS) to the NRTIs didanosine, emtricitabine, and lamivudine (one viral isolate also developed abacavir RS). Virus from Patient-1 and Patient-2 also selected treatment-emergent PI mutations. The 
Table 2. Virologic failure population: baseline characteristics.

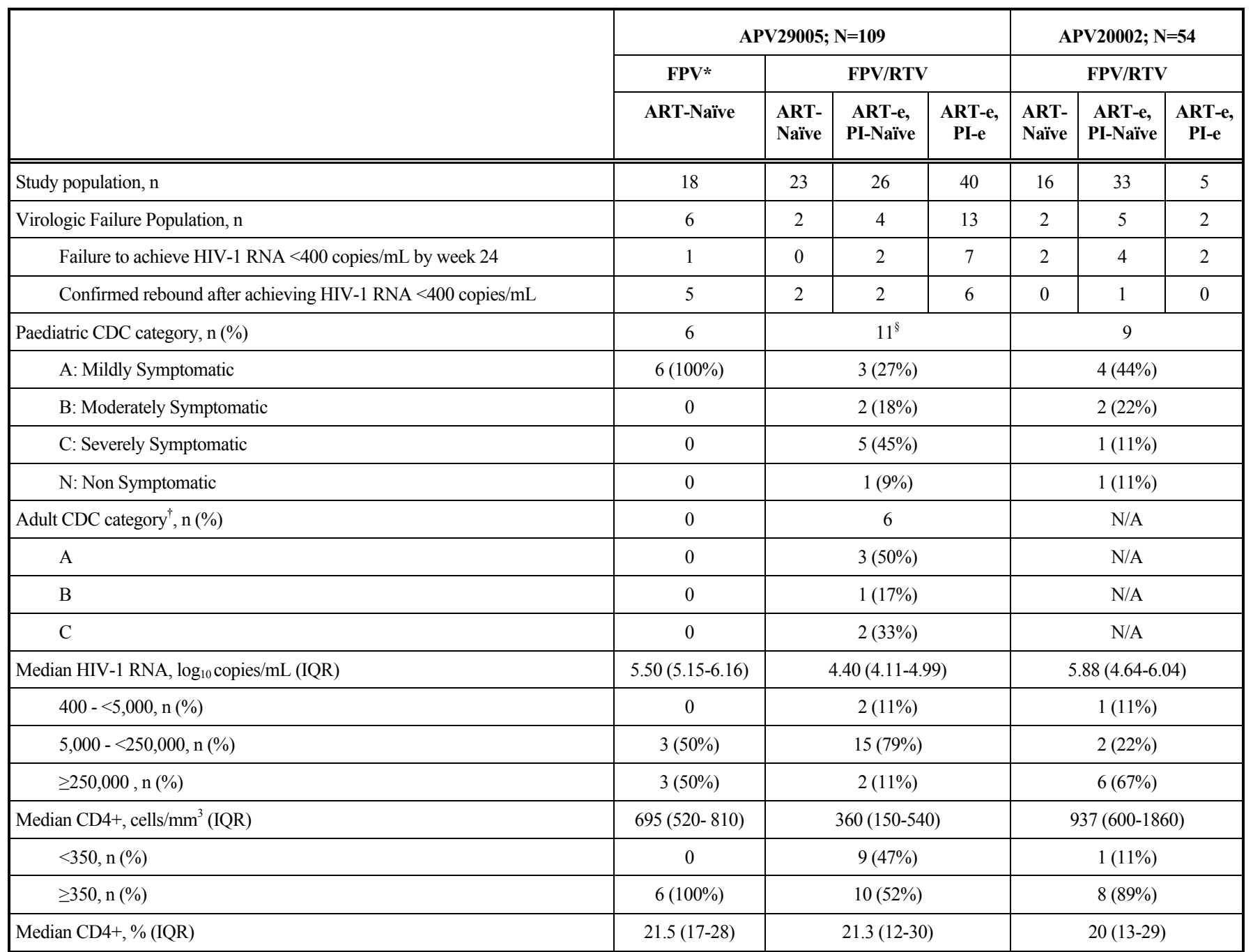

$\mathrm{ART}=$ antiretroviral therapy; e $=$ experienced; FPV = fosamprenavir; IQR = interquartile range; N/A = not applicable; PI = protease inhibitor; RTV = ritonavir; VF = virologic failure.

*There were also two ART-experienced patients taking unboosted FPV in APV29005, but neither met the criteria for virologic failure.

$\S$ CDC classification results were not available for 2 patients.

${ }^{\dagger}$ Adult CDC classification was used for patients $\geq 13$ years of age.

major PI treatment-emergent mutations selected at VF in virus from Patient-1 included M46M/L, I50I/V, I54I/L, and Q58Q/E, while virus from Patient-2 selected the V82V/A mutation. Virus from both patients had treatment-emergent RS to FPV.

Four patients receiving FPV/RTV-containing regimens had virus with treatment-emergent mutations and/or RS at VF. ART-experienced Patient-4 selected a treatmentemergent RT mutation $\mathrm{M} 184 \mathrm{~V}$ at $\mathrm{VF}$ and the virus developed concomitant RS to emtricitabine/lamivudine. Patient-5 had no reported prior PI exposure, but at baseline had multiple major viral PI mutations and RS to 5 PIs. At $\mathrm{VF}$, the following major treatment-emergent PI mutations or mutation mixtures were detected: M46M/I, I50I/V, $\mathrm{I} 54 \mathrm{I} / \mathrm{M} / \mathrm{V}$, and I84I/V, and the virus developed FPV RS. Patient-6 was PI-experienced and at baseline, this patient's virus had major PI mutations and had RS to 5 PIs. The patient met VF criteria at week 24, at which time the major viral PI treatment-emergent mutations I50I/V, I54I/M, and V82F/I were detected. This virus also acquired RS to FPV. Patient-7 had prior NRTI, NNRTI and PI exposure and at baseline virus from this patient had RS to all 3 classes of drug. This patient never achieved virologic suppression and VF criteria were met at week 24. While no treatmentemergent mutations were detected, there was a slight increase in the fold-change (FC) resistance to the drug didanosine (ddI) to above the susceptibility cut-off (baseline $\mathrm{FC}=1.3 ; \mathrm{FC}$ at $\mathrm{VF}=1.39$ ). One additional ART-experienced patient with prior PI exposure met VF at week 48. At baseline, virus from this patient had both NRTI and nonNRTI mutations. Genotyping and phenotyping results were obtained at week 60 . There were no treatment-emergent NRTI or PI mutations although a treatment-emergent NNRTI polymorphism V179V/D/E was detected. This change did not result in any change in NNRTI susceptibility and the response profile is not included in the supplementary figures. 
Table 3. Virologic failure population: treatment-emergent HIV-1 mutations and reduced HIV drug susceptibility.

\begin{tabular}{|c|c|c|c|c|c|c|c|}
\hline & \multicolumn{4}{|c|}{ APV29005 } & \multirow{2}{*}{\multicolumn{3}{|c|}{$\begin{array}{c}\text { APV20002 } \\
\text { FPV/RTV }\end{array}$}} \\
\hline & \multirow{2}{*}{$\frac{\text { FPV }}{\text { ART-Naïve }}$} & \multicolumn{3}{|c|}{ FPV/RTV } & & & \\
\hline & & ART-Naïve & $\begin{array}{l}\text { ART-e, } \\
\text { PI-Naïve }\end{array}$ & $\begin{array}{c}\text { ART-e, } \\
\text { PI-e }\end{array}$ & ART-Naïve & $\begin{array}{l}\text { ART-e, } \\
\text { PI-Naïve }\end{array}$ & $\begin{array}{c}\text { ART-e, } \\
\text { PI-e }\end{array}$ \\
\hline Virologic failure population, $\mathrm{n}$ & 6 & 2 & 4 & 13 & 2 & 5 & 2 \\
\hline With paired genotypes, $\mathrm{n}$ & 3 & 1 & 3 & 8 & 2 & 3 & 2 \\
\hline \multicolumn{8}{|c|}{ Treatment-emergent genotypic mutations, $\mathrm{n}(\%)$} \\
\hline Any NRTI mutation & $3(100 \%)$ & 0 & 0 & $1(13 \%)$ & $1(50 \%)$ & 0 & 0 \\
\hline M184V & $3(100 \%)$ & & & $1(13 \%)$ & $1(50 \%)$ & & \\
\hline Any NNRTI mutation & 0 & 0 & 0 & $1(13 \%)$ & 0 & 0 & $1(50 \%)$ \\
\hline $\mathrm{K} 101 \mathrm{~K} / \mathrm{E}$ & & & & 0 & & & $1(50 \%)$ \\
\hline V179D/E & & & & $1(13 \%)$ & & & 0 \\
\hline Any major PI mutation & $2(67 \%)$ & 0 & $1(33 \%)$ & $1(13 \%)$ & 0 & 0 & 0 \\
\hline $\mathrm{M} 46 \mathrm{M} / \mathrm{I}$ or $\mathrm{M} / \mathrm{L}$ & $1(33 \%)$ & & $1(33 \%)$ & 0 & & & \\
\hline $\mathrm{I} 50 \mathrm{I} / \mathrm{V}$ & $1(33 \%)$ & & $1(33 \%)$ & $1(13 \%)$ & & & \\
\hline $\mathrm{I} 54 \mathrm{I} / \mathrm{L}, \mathrm{I} / \mathrm{M}$ or $\mathrm{I} / \mathrm{M} / \mathrm{V}$ & $1(33 \%)$ & & $1(33 \%)$ & $1(13 \%)$ & & & \\
\hline Q58Q/E & $1(33 \%)$ & & 0 & 0 & & & \\
\hline $\mathrm{V} 82 \mathrm{~A} / \mathrm{V}$ or $\mathrm{F} / \mathrm{I}$ & $1(33 \%)$ & & 0 & $1(13 \%)$ & & & \\
\hline $\mathrm{I} 84 \mathrm{I} / \mathrm{V}$ & 0 & & $1(33 \%)$ & 0 & & & \\
\hline Any minor PI mutation & $2(67 \%)$ & 0 & $1(33 \%)$ & $1(13 \%)$ & $2(100 \%)$ & 0 & $1(50 \%)$ \\
\hline \multicolumn{8}{|c|}{ Treatment-emergent reduced drug susceptibility, $\mathrm{n}(\%)$} \\
\hline Any NRTI & $3(100 \%)$ & 0 & 0 & $2(25 \%)$ & $1(50 \%)$ & 0 & 0 \\
\hline Abacavir & $1(33 \%)$ & & & 0 & 0 & & \\
\hline Didanosine & $3(100 \%)$ & & & $1(13 \%)$ & 0 & & \\
\hline Emtricitabine & $3(100 \%)$ & & & $1(13 \%)$ & $1(50 \%)$ & & \\
\hline Lamivudine & $3(100 \%)$ & & & $1(13 \%)$ & $1(50 \%)$ & & \\
\hline Any PI & $2(67 \%)$ & 0 & $1(33 \%)$ & $1(13 \%)$ & 0 & 0 & $1(50 \%)$ \\
\hline Fosamprenavir $(2 \mathrm{FC}) *$ & $2(67 \%)$ & & N/A & N/A & & & N/A \\
\hline Fosamprenavir (4 FC)* & N/A & & $1(33 \%)$ & $1(13 \%)$ & & & $1(50 \%)$ \\
\hline Ritonavir & $2(67 \%)$ & & 0 & 0 & & & $1(50 \%)$ \\
\hline
\end{tabular}

$\mathrm{ART}=$ antiretroviral therapy; $\mathrm{e}=$ experienced; $\mathrm{FC}=$ fold change; $\mathrm{FPV}=$ fosamprenavir; $\mathrm{N} / \mathrm{A}=$ not applicable; NNRTI $=$ non-nucleoside reverse transcriptase inhibitor; $\mathrm{NRTI}=$ nucleoside reverse transcriptase inhibitor; $\mathrm{PI}=$ protease inhibitor; $\mathrm{RTV}=$ ritonavir.

$*$ Reduced susceptibility FC cut-off for unboosted FPV $=2$; FC cut-off for FPV/RTV $=4$.

\section{Darunavir Cross-Resistance in the VF Population in Study APV29005}

FPV and the PI darunavir can both select for overlapping viral resistance-associated mutations. Darunavir susceptibility data was available at the VF timepoints for virus from 11 patients. No RS to darunavir was observed, although treatment-emergent major PI mutations associated with FPV and darunavir resistance were observed in virus from 4 patients at VF (Supplementary Figs. 1, 2).

\section{Treatment-Emergent HIV-1 Mutations and RS in the VF Population in Study APV20002}

In APV20002, 7/9 VF patients (78\%) had paired genotypes available at baseline and at VF. Of these, 2 were from patients who were ART-naïve at baseline, 3 were ARTexperienced but PI-naïve patients and 2 patients were ARTexperienced with prior PI-exposure. Virus from 3 patients developed treatment-emergent mutations (Table 3 ).

Virus from ART-naïve Patient-1 had a treatmentemergent minor PI mutation at VF which did not result in any change in drug susceptibility (Supplementary Fig. 3). Virus from ART-naïve Patient-2 selected the NRTI M184V at VF, with concomitant RS to the NRTIs emtricitabine and lamivudine. Patient-3 was ART-experienced with prior PI exposure. At baseline the virus from Patient-3 had NRTI and major and minor PI mutations, resulting in RS to several NRTIs and multiple PIs. Virologic suppression was not achieved by week 24 (the VF timepoint). Although NNRTIs were not used in this study, a minor NNRTI mutation 
$\mathrm{K} 101 \mathrm{~K} / \mathrm{E}$, as well as the 2 minor PI mutations L10F and $\mathrm{L} 33 \mathrm{~F}$ emerged at $\mathrm{VF}$ and $\mathrm{RS}$ to FPV was observed.

\section{Darunavir Cross-Resistance in the VF Population in Study APV20002}

Eight of the nine patients in APV20002 with VF had darunavir susceptibility results obtained from virus isolated at the VF timepoint. None had RS to darunavir. Of the PI mutations associated with darunavir drug resistance, only the minor mutation $\mathrm{L} 33 \mathrm{~F}$ or an $\mathrm{L} 33 \mathrm{~L} / \mathrm{F}$ mutation mixture was treatment-emergent in virus from 2 patients at VF.

\section{DISCUSSION}

Although PIs, NNRTIs, and integrase inhibitors continue to be key components of both first and second-line ART regimens, the lack of pediatric formulations as well as crossresistance that can occur within a drug class continue to limit the options available for children. Several protease inhibitors are now approved for use in HIV-infected children in numerous countries. FPV was recently approved for use in children aged at least 4 weeks to 18 years in the United States [11].

The virology analyses presented here from the APV29005 and APV20002 studies together encompass the widest age range (4 weeks to 18 year age), and the largest number of FPV and FPV/RTV-treated pediatric patients from prospective clinical trials published to date. The overall findings from the APV29005 and APV20002 studies of low rates of VF and low rates of selection for PI mutations at VF and FPV RS, especially in the FPV/RTV treated populations, are similar to the limited data available for two small pediatric studies with FPV/RTV, where low rates of VF and selection for resistance mutations were observed $[6,7]$. In one, 7 ART-experienced, HIV-infected children whose virus already contained NRTI resistance mutations were switched to dual protease therapy with FPV/RTV and ATV. All 7 children became virologically suppressed shortly after therapy initiation and remained suppressed to $<50$ copies $/ \mathrm{mL}$ through 42 months on therapy [6]. In a second study, HIVinfected, ART-naïve and ART-experienced children were treated with FPV/RTV (19/20) or FPV-containing regimens $(1 / 20)$, and $14 / 20(70 \%)$ children achieved or maintained virologic suppression. One FPV/RTV-treated, ARTexperienced patient stopped therapy due to high FPV resistance. This patient's virus had the major PI mutations M46L, V82A and L90M at baseline, and the I54L mutation emerged during therapy [7].

In the adult studies (SOLO and KLEAN), in which ARTnaïve patients were treated with FPV/RTV plus abacavir/lamivudine through 48 weeks, virus with treatmentemergent PI mutations was similarly rare and of a similar nature to the treatment-emergent PI mutations observed in APV29005 and APV20002. Of 756 subjects enrolled in both the KLEAN and SOLO studies, a total of 55 patients met protocol-defined VF and 9 had virus with any treatmentemergent PI mutation $[2,3,12]$. In ART-naïve adults treated with unboosted FPV plus NRTIs (the NEAT study), a higher proportion of enrolled subjects met VF criteria (30/166 subjects), of these 10 had virus with any PI treatmentemergent mutation [1].
Comparisons for ART and PI-experienced children are more complicated because of treatment history differences and variability in the types and number of resistance mutation that may have been selected and/or archived during earlier VF. Data from treatment-experienced adults from the CONTEXT and TRIAD studies demonstrated that mutations selected during prior PI treatment can impact response to subsequent FPV treatment [4, 5]. In APV29005 and APV20002, almost half of the ART-experienced children who met VF criteria received regimens that contained $\leq 2$ phenotypically active drugs at baseline. While 23\% (24/104) of the combined ART-experienced population from both studies met VF criteria, either by failing to achieve virologic suppression by week 24 or though virologic rebound through 48 weeks, virus from only one PI-experienced child selected for any major treatment-emergent PI mutation.

Although the only other prior FPV/RTV pediatric studies have already been described [6,7], 2 additional pediatric studies that include resistance evaluations in second-line treatment regimens with other PIs are available for comparison [13, 14]. In one, $30 \%$ of ART-experienced children switched to a darunavir/ritonavir-based regimen aged 6-17 years experienced VF $\left(<1 \log _{10}\right.$ HIV-1 RNA reduction) through 48 weeks. Treatment-emergent HIV-1 protease mutations were seen in $>10 \%$ of children experiencing virologic rebound. A diminished virologic response rate $(<75 \%$ of overall response) was seen in children with $>2$ darunavir-associated baseline HIV-1 resistance mutations [13]. In another study, 108 Ugandan treatment-experienced children whose virus at baseline contained the RT mutation M184V were switched to PIcontaining regimens. The HIV-1 RNA $<400$ copies $/ \mathrm{mL}$ response rates were $79.6 \%$ and $84.5 \%$ at 24 and 48 weeks, respectively [14]. Although viral genotyping was not performed at VF, the presence of another mutation at time of switch to second line therapy $(\mathrm{K} 103 \mathrm{~N})$ was significantly associated $(\mathrm{p}=0.039)$ with poor ART adherence [14].

Subtype data was not available for comparison from the other FPV studies, however, within the APV29005 and APV20002 studies, no qualitative difference between subtypes was observed with regard to meeting VF criteria, or in the types of PI mutations that were treatment-emergent, and the treatment-emergent mutations were consistent with what has been previously observed in adults treated with FPV or FPV/RTV-containing regimens.

Limitations of this analysis include the moderate sample size and variability among patients: 159 children across 2 studies with $2 \mathrm{FPV}$ dosing regimens, 3 ART experience levels, multiple HIV-1 subtypes and range of ages at study entry, and the inability to obtain genotype results from virus from all patients with VF, since $35 \%$ of VF patients lacked paired genotype. Both studies included a significant proportion of subjects with prior ART exposure and baseline drug susceptibility and viral mutation results suggest that for many previously treated subjects, their prior ART exposure resulted in selection for mutations that could have lessened their ability to have a fully effective regimen at the study start. Since population genotyping cannot detect low abundance viral quasi-species, it is possible that prior ART exposure resulted in archiving of additional drug resistant 
variants that were undetectable at baseline but emerged under drug selection pressure and were detected at VF.

In conclusion, the overall types and patterns of treatmentemergent, viral resistance-associated mutations from children receiving FPV or FPV/RTV-based regimens at VF were similar to the resistance profiles seen in adults at VF receiving FPV or FPV/RTV-based regimens. The overall VF rate of $21 \% \quad(34 / 163$ patients) through 48 weeks was relatively low, given the high proportion of ARTexperienced children $(71 \%)$ enrolled in these two studies. Treatment-emergent mutations were most common in children receiving unboosted FPV. Treatment-emergent NRTI, NNRTI and PI mutations were observed in virus from 10 children who experienced VF in the two studies, although some had only treatment-emergent minor mutations. Emergence of drug resistance to FPV was uncommon, with virus from only 2 ART-naïve children receiving FPV and 2 ART-experienced children on FPV/RTV developing FPV RS through 48 weeks. Cross resistance to darunavir was not observed in any of the VF viral isolates, including those that had RS to FPV. These virology results provide additional support for the use of FPV-containing ART regimens in HIV-infected children.

\section{CONFLICT OF INTEREST}

L.R. is an employee of ViiV Healthcare. M.C. received support from a grant from ViiV Healthcare and also payment from Abbott Laboratories for lectures, including service on speakers' bureaus. H.C. and E.V. have no funding or conflicts of interest to declare. N.G., J.S, and K.C. are employees of GlaxoSmithKline.

\section{ACKNOWLEDGEMENTS}

The authors would like to thank all of the study patients and their families, the study investigators and their staff, and the extended GSK/ViiV study team, especially Tony Thompson, Terri Becom, Deborah Copeland, and Don Shanahan, and to Katherine A. DeBruin for writing contributions.

This resistance analysis was presented at the $19^{\text {th }}$ International AIDS Conference, July 22-27, 2012, Washington, DC.

This study was sponsored and funded by ViiV Healthcare; GlaxoSmithKline is responsible for study implementation.

\section{SUPPLEMENTARY MATERIAL}

Supplementary material is available on the publisher's website along with the published article.

\section{REFERENCES}

[1] Rodriguez-French A, Boghossian J, Gray GE, et al. The NEAT study: a 48-week open-label study to compare the antiviral efficacy and safety of GW433908 versus nelfinavir in antiretroviral therapynaïve HIV-1-infected patients. J Acquir Immune Defic Syndr 2004; 35: 22-32.

[2] Gathe JC, Ive P, Wood R, et al. SOLO: 48-week efficacy and safety comparison of once-daily fosamprenavir /ritonavir versus twice-daily nelfinavir in naive HIV-1-infected patients. AIDS 2004; 18: 1529-37.

[3] Eron J, Yeni P, Gathe J, et al. The KLEAN study of fosamprenavirritonavir versus lopinavir-ritonavir, each in combination with abacavir-lamivudine, for initial treatment of HIV infection over 48 weeks: a randomised non-inferiority trial. Lancet 2006; 368: 476782 .

[4] Molina JM, Ait-Khaled M, Rinaldi R, et al. Fosamprenavir/ritonavir in advanced HIV disease (TRIAD): a randomized study of highdose, dual-boosted or standard dose fosamprenavir/ritonavir in HIV-1-infected patients with antiretroviral resistance. J Antimicrob Chemother 2009; 64: 398-410.

[5] Marcelin AG, Flandre P, Molina JM, et al. Genotypic resistance analysis of the virological response to fosamprenavir-ritonavir in protease inhibitor-experienced patients in CONTEXT and TRIAD clinical trials. Antimicrob Agents Chemother 2008; 52: 4251-7.

[6] Rusconi S, Giacomet V, Mameli C, et al. Efficacy and safety of a dual boosted protease inhibitor-based regimen, atazanavir and fosamprenavir/ritonavir, against HIV: experience in a pediatric population. BMC Infect Dis 2012; 12:179.

[7] Palladino C, Briz V, Policarpo SN, et al. Long-term efficacy and safety of fosamprenavir in human immunodeficiency virus-infected pediatric patients. Pediatr Infect Dis J 2010; 29: 563-6.

[8] Cotton M, Cassim H, Pavía-Ruz N, et al. Pharmacokinetics, safety and antiviral activity of fosamprenavir/ritonavir-containing regimens in HIV infected infants and children 4 week to 2 years of age: 48 week study data. Pediatr Infect Dis J 2014; 33(1): 57-62.

[9] Fortuny C, Duiculescu D, Cheng K, et al. Pharmacokinetics and 48 week safety and antiviral activity of fosamprenavir-containing regimens in HIV-infected 2 to 18 year-old children. Pediatr Infect Dis J 2014; 33(1): 50-6.

[10] Johnson VA, Brun-Vezinet F, Clotet B, et al. Update of the drug resistance mutations in HIV-1: December 2010. Top HIV Med 2010; 18: 156-63.

[11] LEXIVA Label Information; [cited on May 15 2014]. Available at http://www.accessdata.fda.gov/drugsatfda docs/label/2013/021548 s031,022116s015lbl.pdf.

[12] Ross L, Vavro C, Wine B, et al. Fosamprenavir clinical study meta-analysis in ART-naïve subjects: rare occurrence of virologic failure and selection of protease-associated mutations. HIV Clin Trials 2006; 7: 334-8.

[13] Blanche S, Bologna R, Cahn P, et al. Pharmacokinetics, safety and efficacy of darunavir/ritonavir in treatment-experienced children and adolescents. AIDS 2009; 23: 2005-13.

[14] Musiime V, Kaudha E, Kayiwa J, et al. Antiretroviral drug resistance profiles and response to second-line therapy among HIV type 1-infected Ugandan children. AIDS Res Hum Retroviruses 2013; 29: 449-55. 